CAuteurs(es). Cette œuvre, disponible à http://dx.doi.org/10.18162/fp.2020.511, est distribuée sous licence Creative Commons Attribution 4.0 International http://creativecommons.org/licences/by/4.0/deed.fr
Adolphe Adihou

Université de Sherbrooke (Canada)

Cathy Arsenault

Université du Québec à Rimouski (Canada)

\section{Formation à l'enseignement des mathématiques : opérationnalisation et regard des étudiants sur un dispositif de formation}

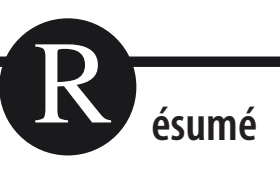

Cette étude descriptive vise à caractériser la démarche personnelle de formation des étudiants pour développer leurs compétences à l'enseignement des mathématiques et à cerner les impacts d'un dispositif de formation. Les analyses ont permis d'identifier les types de connaissances à l'enseignement des mathématiques mobilisées par les étudiants dans leur démarche (Loewenberg Ball, Thames et Phelps, 2008), leurs attentes et satisfactions. Les résultats démontrent que le dispositif favorise le développement d'attitudes professionnelles nourrissant le sentiment d'efficacité personnel (Bandura, 2007) et révèle la nécessité d'articuler les dimensions diagnostique, mathématique, didactique et pratique de la formation à l'enseignement.

Mots-clés

Démarche de formation, dispositif de formation, mathématiques, didactique, articulation.

Abstract

This descriptive study aims to characterize the personal training approach of students to develop their skills in teaching mathematics and to identify the impacts of a training system. The analyses made it possible to identify the types of mathematics teaching knowledge mobilized by the students in their approach (Loewenberg Ball, Thames and Phelps, 2008), their expectations and satisfactions. The results show that the system promotes the development of professional attitudes that foster a sense of personal effectiveness (Bandura, 2007) and reveals the need to articulate the diagnostic, mathematical, didactic and practical dimensions of teacher education.

\section{Keywords}

Training approach, training system, mathematics, didactics, articulation.

\section{Introduction}

Plusieurs chercheurs en didactique des mathématiques (Adihou et Arsenault, 2012; Bednarz et Proulx, 2009; Clivaz, 2016; Coulange et Robert, 2015) tentent de définir la spécificité et la place de la formation mathématique dans les programmes en éducation afin de concevoir des dispositifs assurant le développement des compétences à l'enseignement des mathématiques chez les futurs maitres.

Notre étude vise à caractériser et analyser la démarche dans laquelle des étudiants ${ }^{1}$ inscrits au baccalauréat en éducation préscolaire et en enseignement au primaire (BEPEP) à l'Université du Québec à Rimouski (UQAR) s'inscrivent pour développer leurs compétences à l'enseignement des mathématiques. Nous cherchons à connaître leur rapport aux savoirs mathématiques et les impacts d'un dispositif de formation dans le développement de leurs compétences. L'analyse des attentes et satisfactions des étudiants vise également à bonifier le dispositif.

Cet article présente la problématique menant à cette étude, le cadre de référence et la méthodologie de recherche. Les résultats sont décrits et commentés. En conclusion, nous interrogeons la pertinence, l'efficacité du dispositif et proposons quelques pistes de réflexion pour l'améliorer.

\section{Contexte et problématique}

Un des buts de la recherche en didactique des mathématiques est de mieux comprendre les phénomènes d'enseignement et d'apprentissage en classe de mathématiques, de les examiner et d'identifier ceux caractérisant la discipline enseignée afin de trouver comment agir pour améliorer son enseignement (Conne, 1989). La formation 
à l'enseignement des mathématiques vise à développer chez les futurs maitres les compétences professionnelles leur permettant d'amener les élèves à construire des connaissances mathématiques et à l'outiller dans sa pratique d'enseignement (Robert, 2008). Dans sa thèse de doctorat, Clivaz précise :

La question de l'efficacité de l'enseignement et la nécessité de former et de sélectionner des enseignants pouvant être efficaces ont conduit à distinguer et à catégoriser les connaissances nécessaires à l'enseignement et à construire des outils permettant de tester ces connaissances. (Clivaz, 2011, p. 23)

Ces efficacités, dont parle Clivaz, reposent sur des dispositifs de formation complexes mis en place dans plusieurs institutions de formation initiale à l'enseignement des mathématiques (Lajoie et Tempier, 2019). Au Québec, pour atteindre les objectifs du ministère de l'Éducation concernant cet enseignement (MEQ 2001) et ces niveaux d'efficacité (Clivaz, 2011), plusieurs chercheurs québécois s'intéressent à cette formation en abordant diverses problématiques : le type de mathématiques pour les futurs enseignants (Proulx, Corriveau et Squalli, 2012); leurs difficultés en mathématiques (Adihou et Arsenault, 2012; Adihou, Arsenault et Marchand, 2012; Bednarz et Proulx, 2009); les entrées possibles pour une formation à l'enseignement (DeBlois, 2010; GREFEM, 2012; Lajoie, Maheux, Marchand, Adihou et Bisson, 2012; Proulx, 2019). Leurs travaux ont permis de concevoir différents outils pour évaluer les compétences et répondre aux besoins des étudiants.

Dans ce contexte, I'UQAR propose un dispositif de formation comprenant trois volets : un examen ${ }^{2}$ des compétences en mathématiques visant à informer l'étudiant sur sa compréhension des mathématiques et à susciter une démarche de développement dont il est responsable; une formation mathématique pour remédier aux lacunes en fournissant des outils conceptuels; une formation didactique pour développer des compétences à enseigner les mathématiques au primaire (figure 1).

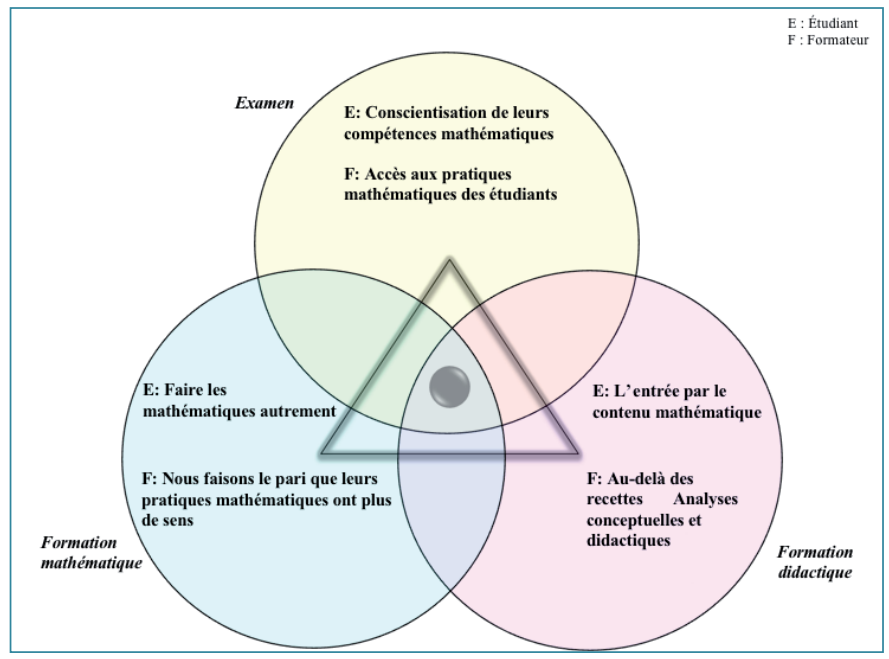

Figure 1

Dispositif de formation à l'enseignement des mathématiques : articulation des trois volets. 
Le dispositif repose sur un cadre théorique éprouvé, favorisant l'articulation des formations mathématique et didactique (Loewenberg Ball, Thames et Phelps, 2008). Effectivement, le travail mathématique réalisé par les étudiants, suite à l'examen, soulève une réflexion sur l'apprentissage des concepts et processus mathématiques, tandis que le travail didactique favorise la poursuite de cette réflexion vers l'enseignement de ceux-ci (Adihou et Arsenault, 2012). En interaction, les connaissances mathématiques et didactiques sont déployées à différents moments et lieux de formation, favorisant leur articulation. Ainsi, pour développer l'ensemble des compétences professionnelles nécessaires à l'enseignement des mathématiques, le travail de l'étudiant s'échelonne sur les quatre années du baccalauréat.

De l'automne 2004 à l'hiver 2016, le suivi des cohortes d'étudiants inscrits à ce dispositif révèle que la moyenne des pourcentages de réussite à l'examen, après quatre ans et plus, atteint 78,4\%. Le taux d'abandon et de cheminement particulier se situe à 18,2\% en moyenne, tandis que 3,4 \% des étudiants n'ayant pas réussi l'examen sont suspendus du programme. Quant aux différentes mesures d'aide, environ $40 \%$ des étudiants y participent pour développer leurs compétences en mathématiques (Adihou et Arsenault, 2012). Que font-ils comme démarche? Quelles sont les caractéristiques de celle-ci et sur quels types de connaissances (Loewenberg Ball et al., 2008) s'appuie-t-elle? Quelles sont leurs attentes et satisfactions au regard de la formation à l'enseignement des mathématiques? Se sentent-ils compétents et prêts à les enseigner?

Les réponses à ces questions permettront de connaître le cheminement professionnel des étudiants inscrits à ce dispositif de formation. Mais au-delà des questions, la pérennité de celui-ci nécessite une approche réflexive visant l'évaluation des composantes et des choix didactiques sur lesquels il repose afin de le bonifier et d'améliorer sa pertinence.

\section{Cadre de référence et objectifs de recherche}

Cette recherche s'appuie sur les travaux traitant de la nature des connaissances mathématiques à l'enseignement, leurs liens et usages en formation (Bednarz, 2012; Clivaz, 2011, 2016; DeBlois, 2010; Lajoie et al., 2012; Loewenberg Ball et al., 2008; Proulx, 2019). Ces études démontrent qu'au-delà des connaissances mathématiques acquises lors du parcours scolaire, les futurs maitres doivent construire d'autres connaissances mathématiques spécifiques à l'enseignement et développer des compétences relatives à celles-ci. Afin de mieux contextualiser les savoirs théoriques et tisser des liens entre les connaissances théoriques et pratiques, ce travail s'effectue en différents lieux de formation.

Des chercheurs (Loewenberg Ball et al., 2008; Shulman, 1986, 1987) identifient des types de connaissances intervenant dans le développement des compétences pour l'enseignement. Shulman (1986) présente trois catégories de connaissances relatives à l'enseignement, qu'il bonifie ensuite en sept catégories, soit : 1) les connaissances du contenu, 2) les connaissances pédagogiques générales, 3) les connaissances du curriculum, 4) les connaissances pédagogiques du contenu, 5) les connaissances des élèves et de leurs caractéristiques, 6) les connaissances du contexte de la classe ou de l'école, 7) les connaissances des finalités, des valeurs, des fondements philosophiques et historiques de l'éducation (Shulman, 1987, cité dans Clivaz, 2011). 
Loewenberg Ball et al. (2008) distinguent différents types de connaissances mathématiques nécessaires pour enseigner cette discipline, qu'ils regroupent en deux grandes catégories : les connaissances du sujet ou de la matière et les connaissances pédagogiques et didactiques. Chacune de ces catégories se subdivise en trois sous-catégories (figure 2).

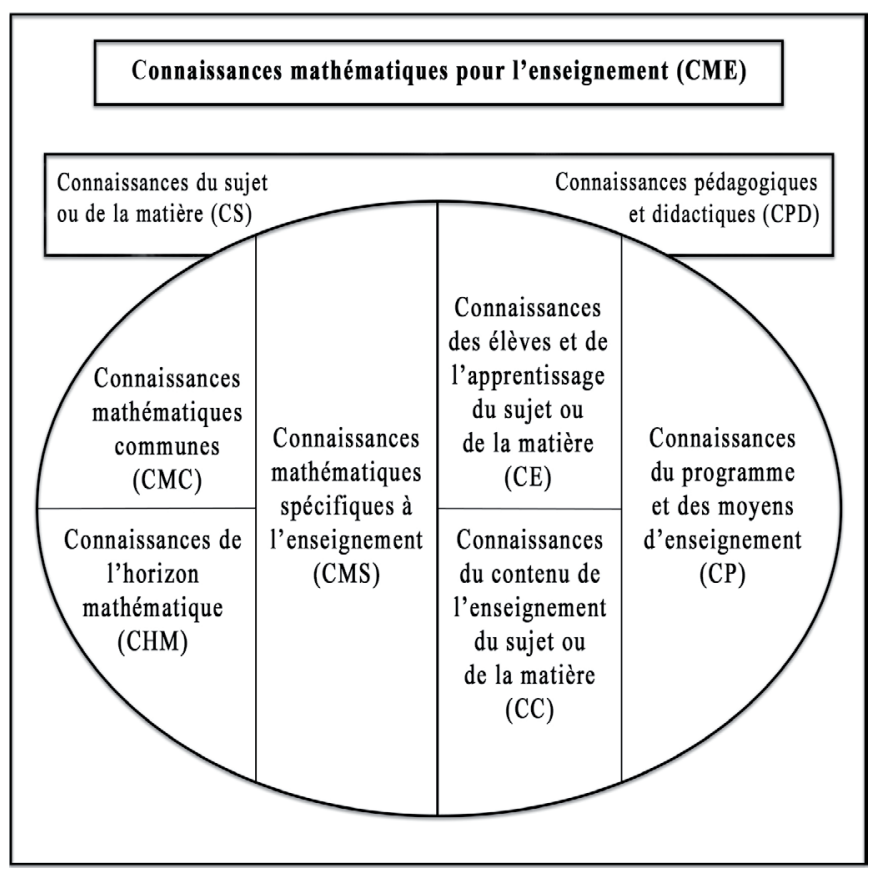

Figure 2

Connaissances mathématiques pour l'enseignement, traduit de Loewenberg Ball et al. (2008).

Les connaissances du sujet ou de la matière regroupent trois types de connaissances. Les connaissances mathématiques communes se composent de techniques et méthodes intervenant dans diverses disciplines faisant appel aux savoirs mathématiques. Elles permettent, entre autres, des applications, des modélisations et l'étude de situations dans un contexte d'analyses de tâches. Les connaissances de l'horizon mathématique favorisent l'articulation et le lien entre les contenus mathématiques. Cellesci permettent de cerner les fondements et bases des contenus, apportant une vision plus large des mathématiques. Les connaissances mathématiques spécifiques à l'enseignement permettent l'enseignement et l'apprentissage des contenus. Ce sont des transpositions de savoirs mathématiques au cœur du processus d'apprentissage. Des erreurs mathématiques en font partie ainsi que des techniques et méthodes de résolution. Les savoirs peuvent être liés à des connaissances "primitives ", mais qui se légitiment par des référents mathématiques.

Les connaissances pédagogiques et didactiques regroupent trois types de connaissances. Elles se composent des connaissances de l'élève et de l'apprentissage du sujet ou de la matière. Celles-ci interviennent dans la compréhension et les processus d'acquisition des concepts mathématiques. Elles permettent de cerner les spécificités de l'élève relativement aux concepts, à la nature des erreurs et procédures mathématiques. Elles aident à comprendre les démarches, l'usage des contenus mathématiques et 
les régularités en termes de concepts. Servant de références pour diverses analyses (conceptuelles, $a$ priori et a posteriori), elles sont utiles pour anticiper les procédures, les erreurs et mettre en évidence les fausses conceptions des élèves. Les connaissances du contenu de l'enseignement du sujet ou de la matière constituent les connaissances mathématiques auxquelles l'enseignant se réfère pour planifier les situations d'enseignement-apprentissage. Celles-ci favorisent le choix des activités de préparation, de réalisation, d'objectivation et de réinvestissement. Autrement dit, elles interviennent dans les situations d'actions et participent au processus de dévolution et d'institutionnalisation (Brousseau, 1998). Enfin, les connaissances du programme et des moyens d'enseignement se composent des connaissances sur les contenus des programmes et curricula. Ces savoirs à enseigner sont destinés à l'enseignement et l'apprentissage des concepts mathématiques à différents niveaux et cycles. Elles font aussi référence à des connaissances liées aux supports matériels.

Dans le contexte de la formation à l'enseignement, ces types de connaissances s'opérationnalisent à travers des dispositifs complexes (Lajoie et Tempier, 2019) se définissant comme :

[...] une organisation de ressources (humaines, pédagogiques, matérielles, etc.) au service d'une action finalisée. C'est une construction sociale qui, en jouant des contraintes et de la variété des ressources, agence des situations susceptibles d'entrer en résonance avec les dispositions des personnes en formation. (Carré, 2009, p. 10)

Les éléments de cette définition font écho aux choix effectués dans la conception du dispositif de formation à l'enseignement des mathématiques à l'UQAR. Les connaissances mathématiques caractérisées par Loewenberg Ball et al. (2008) prennent place dans chacune des composantes de celui-ci. Effectivement, l'étudiant démontre son niveau de maitrise des connaissances mathématiques pour l'enseignement $(C S)^{3}$ dans le cadre de l'examen.En s'engageant dans une démarche personnelle de développement des compétences en mathématiques, les connaissances sont activées dans la formation mathématique en vue de les approfondir $(C S)$. Celles-ci sont convoquées dans la formation didactique afin de développer des compétences professionnelles liées au métier d'enseignant (CPD). Si les connaissances mathématiques sont des outils visant le développement professionnel de l'enseignant, les étudiants se sentent-ils prêts à enseigner à la fin de leur cursus? Cette question renvoie au sentiment d'efficacité personnelle (SEP) se définissant par la croyance qu'un individu a en sa capacité à réaliser une tâche, un apprentissage, un défi avec succès, le motivant à s'engager dans l'action et à persévérer pour atteindre son objectif (Bandura, 2007). Le SEP au regard de l'enseignement des mathématiques et la démarche de développement des compétences en mathématiques sont donc interdépendants.

\section{Méthodologie}

Cette étude descriptive porte sur les caractéristiques de la démarche de formation à l'enseignement des mathématiques entreprise par les futurs maitres, leurs attentes et satisfactions concernant le dispositif de formation ainsi que leur SEP au regard de l'enseignement des mathématiques. 


\section{Échantillon}

Les participants sont des étudiants universitaires, inscrits au BEPEP à l'UQAR. L'échantillon, composé d'étudiants en quatrième année de formation, est constitué à l'aide d'un échantillonnage proximal non probabiliste de type volontaire $(\mathrm{n}=54)$. L'échantillon complet est formé en majorité de femmes $(\mathrm{n}=150)$. Parmi les 54 répondants, deux ont un diplôme d'études universitaires. Tous les autres ont un diplôme d'études collégiales. L'âge des répondants varie de 18 ans à plus de 33 ans (tableau 1).

\section{Tableau 1}

Profil des répondants selon l'âge.

\begin{tabular}{l|c}
\hline \multicolumn{1}{c|}{ Catégories d'âge } & Nombre d'étudiants \\
\hline 18 à 22 ans & 17 \\
\hline 23 à 27 ans & 31 \\
\hline 28 à 32 ans & 3 \\
\hline 33 ans et plus & 3 \\
\hline Nombre total d'étudiants & 54 \\
\hline
\end{tabular}

\section{Déroulement}

L'enquête s'est déroulée à l'hiver 2013 et 2014 dans le cadre d'un cours obligatoire du programme de formation. Un questionnaire fut remis aux étudiants de deux groupes-classes. Le fait de le remplir et le remettre constituait un consentement à la recherche.

\section{Outil de collecte de données}

Le questionnaire comporte trois parties. La première permet de recueillir des données sociodémographiques; la seconde, composée de 20 questions à degré d'accord, a trait aux attentes et satisfactions des étudiants au regard des mathématiques, au SEP et à la formation à l'enseignement des mathématiques. Léchelle de réponse, de type Likert, présente quatre niveaux. La dernière partie compte six questions ouvertes à court développement visant à obtenir plus d'information liée à la problématique. Les 26 questions (Annexe 1) sont classées en quatre groupements selon les connaissances de Loewenberg Ball et al. (2008) et nos catégories initiales. Ces dernières renvoient aussi à des variables relatives au SEP (Bandura, 2007), c'est-à-dire l'affectivité, la confiance en soi, la motivation au regard des mathématiques. Ainsi, les questions touchant les catégories Rapport aux mathématiques et Maitrise des contenus permettent de recueillir des données sur le SEP (tableau 2). 


\section{Tableau 2}

Catégorisation des questions destinées aux étudiants de quatrième année.

\begin{tabular}{|c|c|c|c|}
\hline $\begin{array}{l}\text { Catégories de Loewenberg Ball et } \\
\text { al. (2008) }\end{array}$ & Numéro des questions & $\begin{array}{l}\text { Nombre de } \\
\text { questions }\end{array}$ & Catégories initiales \\
\hline $\begin{array}{l}\text { Connaissance du sujet ou de la } \\
\text { matière (CS) }\end{array}$ & $\begin{array}{l}\text { Q17-Q19 } \\
\text { Q25 }\end{array}$ & 3 & $\begin{array}{l}\text { Formation mathématique } \\
\text { Maitrise des contenus }\end{array}$ \\
\hline $\begin{array}{l}\text { Connaissances mathématiques } \\
\text { communes (CMC) et connaissances } \\
\text { de l'horizon mathématique (CHM) }\end{array}$ & $\begin{array}{l}Q 1-Q 2-06 \\
\text { Q5 } \\
\text { Q10-Q11-Q22 } \\
\text { Q21-Q23 }\end{array}$ & 9 & $\begin{array}{l}\text { Rapport aux mathématiques } \\
\text { Contenu mathématique } \\
\text { Maitrise des contenus } \\
\text { Formation mathématique }\end{array}$ \\
\hline $\begin{array}{l}\text { Connaissances mathématiques } \\
\text { spécifiques à l'enseignement (CMS) } \\
\text { et connaissances pédagogiques et } \\
\text { didactiques (CPD) }\end{array}$ & $\begin{array}{l}\text { Q4-Q9 } \\
\text { Q7 } \\
\text { Q8 } \\
\text { Q13 }\end{array}$ & 5 & $\begin{array}{l}\text { Rapport aux mathématiques } \\
\text { Contenu mathématique } \\
\text { Formation mathématique } \\
\text { Maitrise des contenus }\end{array}$ \\
\hline Toutes les catégories & $\begin{array}{l}Q 3-014-024 \\
Q 12-020-026 \\
Q 15 \\
Q 16-018\end{array}$ & 9 & $\begin{array}{l}\text { Contenu mathématique } \\
\text { Maitrise des contenus } \\
\text { Rapport aux mathématiques } \\
\text { Formation mathématique }\end{array}$ \\
\hline
\end{tabular}

\section{Méthode d'analyse des données}

Le traitement des données s'effectue en deux temps. D'abord, les données sociodémographiques et énoncés à degré d'accord sont compilés dans un fichier Excel afin de calculer la fréquence des types de réponses pour chaque question. Ensuite, les questions à court développement sont traitées en procédant à une analyse de contenu des réponses obtenues par un découpage et un classement en unité de sens, en fonction des catégories dégagées au fur et à mesure de l'analyse (Miles et Huberman, 2007; Mucchielli, 2006). Il s'agit, entre autres, des critères de jugement des étudiants au regard de la formation à l'enseignement des mathématiques et de leur démarche personnelle de formation. Pour chaque catégorie, non exclusive, la fréquence est calculée en utilisant le nombre de répondants par catégorie par rapport à l'ensemble des répondants. Ces fréquences et l'analyse de contenu permettent de tirer des conclusions quant à leurs attentes, satisfactions, moyens et démarches.

Bien que l'étude fût réalisée en 2013-2014, les données sont toujours pertinentes. En effet, la problématique de la maitrise des contenus mathématiques par les futurs maitres est encore une préoccupation des formateurs. Les offres de formations en mathématiques et les objectifs du dispositif demeurent les mêmes pour l'UQAR. Par ailleurs, les cohortes d'étudiants ont des caractéristiques semblables au regard de leur âge et parcours académique. Dans ce sens, les attentes et satisfactions des étudiants ainsi que la caractérisation explicite de leur démarche aideront à bonifier la formation mathématique existante. 


\section{Résultats}

L'analyse des données nous permet d'apporter des réponses aux différentes questions formulées dans la problématique. En lien avec les types de connaissances de Loewenberg Ball et al. (2008), nous caractérisons la démarche de développement des compétences en mathématiques des étudiants, puis nous discutons de leur niveau de satisfaction au regard du travail accompli et de la formation reçue ainsi que de leur SEP comme futur enseignant.

\section{Les démarches des étudiants pour développer leurs compétences en mathématiques}

Selon notre enquête, 50,5 \% des répondants choisissent une ou des mesures d'aide offertes par l'UQAR pour développer leurs compétences en mathématiques (CMC, CHM). Les étudiants privilégient les mesures de courte durée, plus souples au regard de leur agenda. La consultation de leur examen $(42,6 \%)$, la participation aux ateliers $(39,7 \%)$ et l'exercisation $(31,0 \%)$ sont les mesures les plus sollicitées (figure 3).

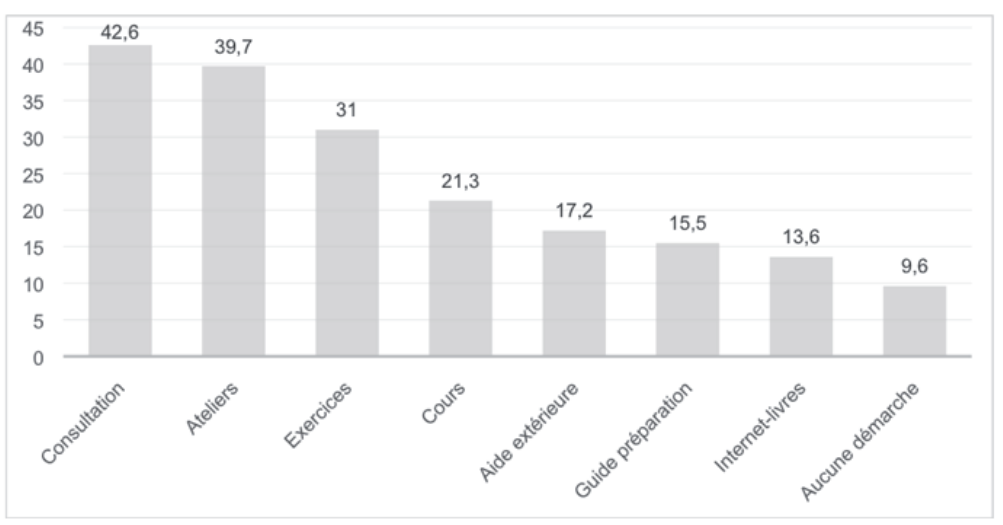

Figure 3

Pourcentages des différentes mesures d'aide choisies par les étudiants.

La consultation de l'examen se déroule au centre d'aide à la réussite (CAR) après une passation. L'étudiant, accompagné de la conseillère en mathématiques, révise ses réponses aux questions, prenant ainsi conscience de ses erreurs et difficultés. Cette étape importante de la démarche permet de choisir les mesures d'aide appropriées. Pour certains étudiants, plusieurs passations s'avèrent nécessaires pour réussir l'examen (tableau 3). Entre chacune d'elles, ils doivent poursuivre leur démarche afin de parfaire leurs connaissances mathématiques. 
Tableau 3

Nombre de passations nécessaires par les étudiants pour la réussite de l'examen.

\begin{tabular}{l|c}
\hline \multicolumn{1}{c|}{ Nombre de passations à l'examen $(P)$} & Nombre d'étudiants \\
\hline 1 passation & 18 \\
\hline 2 passations -1 reprise & 20 \\
\hline 3 passations -2 reprises & 7 \\
\hline 4 passations -3 reprises & 6 \\
\hline 5 passations -4 reprises & 2 \\
\hline 6 passations -5 reprises & 1 \\
\hline Nombre total d'étudiants & 54 \\
\hline
\end{tabular}

Concernant les exercices, les étudiants choisissent différentes approches. Par exemple, l'étudiant ÉL34$2 \mathrm{P}^{4}$ précise qu'il travaille avec un petit groupe d'étudiants : "Je me fais expliquer les exercices que je ne comprends pas et ensuite j’explique à quelqu'un ma comprébension. Je l'enseigne à mon tour». Quant à l'étudiant ÉL23-3P, il préfère travailler seul : "J'ai acheté les différents cabiers d'exercices que le CAR proposait et j'ai fait les exercices. J'ai bien révisé les différentes notions ». Par ailleurs, le guide de préparation à l'examen $(15,5 \%)$ ou la consultation d'ouvrages mathématiques $(13,6 \%)$ représentent aussi des façons de faire des exercices individuellement. Lorsque ces mesures nont pas permis aux étudiants de réussir l'examen de mathématiques, les cours de mathématiques (21,3\%), l'aide extérieure à l'UQAR, par exemple des cours privés $(17,2 \%)$, sont d'autres moyens envisagés. Parmi les répondants affirmant n'avoir fait aucune démarche $(9,6 \%)$, plusieurs d'entre eux réussissent l'examen à la première passation et poursuivent leur formation dans les cours de didactique des mathématiques du programme $(C P D)$.

Voici deux démarches décrites par les étudiants ÉL25-3P et ÉR16-4P démontrant un engagement dans leur formation mathématique $(C M C$ et $C H M)$.

J'ai rencontré la conseillère en mathématique afin de voir le diagnostic de ma première passation. Je lui ai demandé des conseils. J'ai aussi assisté aux ateliers offerts. J'ai réservé des périodes de temps dans mon horaire pour étudier et travailler dans le guide de préparation à l'examen. J'ai fait preuve d'autodidaxie pour réussir l'examen (je gardais en banque les notions plus difficiles).J'ai rencontré la conseillère en mathématiques pour clarifier certaines notions plus difficiles.

Suite à ma première passation de l'examen, j’ai décidé de m’inscrire au cours Savoirs mathématiques ( 3 crédits) et j'ai pris des cours privés. Ces derniers m'aidaient à comprendre les savoirs mathématiques. Après ma deuxième passation, je me suis inscrite au cours de mathématiques de 1 crédit. J'ai assisté à plusieurs ateliers offerts par le CAR. J'ai aussi eu des cours privés une à deux fois/semaine, et ce, pendant 4 mois.

La démarche dans laquelle s'engagent les étudiants pour approfondir leurs connaissances mathématiques $(C M C$ et $C H M)$ comporte donc quelques étapes, combinant deux à trois mesures d'aide. Elle se caractérise par une activité préparatoire à l'examen (ateliers mathématiques, exercisation et guide de 
préparation à l'examen avec l'aide du CAR), la passation de l'examen et ensuite, des activités pour surmonter les difficultés rencontrées (exercisation, cours de mathématiques). Quels sont les apports du travail accompli au regard de leur formation à l'enseignement des mathématiques?

\section{Les apports du dispositif de formation à l'enseignement des mathématiques}

Le travail réalisé par les étudiants au cours des quatre années de formation leur permet de mieux comprendre l'importance accordée à la maitrise des contenus mathématiques $(C S)$ et de profiter davantage de la formation didactique (CPD). À cet effet, l'étudiant ÉL35-2P souligne : "Il faut se prendre en main dès le début, car la comprébension des concepts est préalable aux cours de didactique et une excellente maitrise de ces concepts est nécessaire pour enseigner » (CS et CPD), tandis que l'étudiant ÉL92P ajoute : "La maitrise des contenus permet de mieux comprendre la didactique. Selon moi, l'inverse est difficile» (CS et CPD). Pour d'autres (7,8\%), la révision des notions et concepts mathématiques $(C M C$ et $C H M)$ se révèle l'apport le plus significatif, comme en témoigne l'étudiant ÉL21-4P : «J'ai énormément travaillé mes compétences en mathématiques! Je me suis dépassé! Je crois qu'aujourd'hui, j’aime beaucoup plus les mathématiques puisque je les comprends! Cela a un impact positif sur ma façon d'enseigner les mathématiques»(CS et CPD). L'étudiant ÉL27-2P souligne l'importance du sens des connaissances pour être un meilleur enseignant : "En sachant très bien les notions, il est plus facile d'en comprendre le sens et ainsi de créer des activités déclencheurs plus motivantes et signifiantes aux élèves » (CS et CPD).

Au regard de la démarche de formation, 50 \% des étudiants admettent quelle leur permet d'améliorer leurs compétences en mathématiques $(C M C$ et $C H M)$. Par ailleurs, les étudiants recommandent à leurs pairs de : faire des efforts pour réussir (51\%); demander de l'aide sans hésitation (41,2\%); commencer tôt le travail sur les compétences en mathématiques (21,6\%); travailler leurs difficultés $(17,7 \%)$. L'étudiant ÉL21-4P donne les conseils suivants : "Il ne faut pas perdre une minute pour commencer à étudier les mathématiques. Aller au CAR, regarder la feuille diagnostique et surtout ne pas perdre espoir. Il faut toujours travailler les mathématiques même après avoir réussi l'examen » $(C M C$ et $C H M)$. Si la démarche favorise le développement de compétences en mathématiques, contribue-t-elle au développement du SEP? Les étudiants sont-ils satisfaits de la formation à l'enseignement des mathématiques?

\section{Le SEP et le niveau de satisfaction au regard de la formation}

La majorité des étudiants considèrent qu'ils ont une attitude favorable à l'égard des mathématiques, qu'ils maitrisent l'ensemble des contenus à enseigner et seront d'excellents enseignants. Ainsi, 72,5\% des étudiants ont le sentiment d'être efficace à l'égard de l'enseignement des mathématiques (CMS et $C P D$ ) et 70,4\% ont l'intention de poursuivre leur formation mathématique dans le cadre de la formation continue (CMS et CPD). Selon Bandura (2007), les personnes démontrant un fort SEP s'avèrent plus proactives pour atteindre leurs objectifs. En effet, 92,6 \% des répondants sont d'avis qu'il faut avoir une solide base en mathématiques $(C M C$ et $C H M)$ pour pouvoir les enseigner au primaire ( $C M S$ et $C P D$ ) et 98,2\% reconnaissent l'importance de la formation mathématique (CMC et $C H M)$ dans la formation à l'enseignement $(C M S$ et $C P D)$. En tant que didacticiens, nous apprécions cette ouverture à l'amélioration des compétences à l'enseignement des mathématiques. 
Concernant leur satisfaction à l'égard de la formation reçue, 34,6 \% des étudiants sont satisfaits, tandis que 29,4 \% aimeraient avoir plus de moyens pédagogiques et de matériel d'enseignement-apprentissage à leur disposition (CPD). En effet, l'étudiant ÉL2-1P écrit : "Nous avons besoin de concret, de conseils, de méthodes afin de savoir comment enseigner les mathématiques». Létudiant ÉL37-1P ajoute : "On manque d'activités et de pistes didactiques, on ne sait pas comment partir de l'erreur de l'élève pour le faire progresser. On manque de connaissance sur comment enseigner les stratégies aux élèves en mathématiques». Pour d'autres, l'expérience et l'acquisition de trucs permettraient d'augmenter leur SEP au regard de l'enseignement des mathématiques, tel que le précise l'étudiant ÉR11-3P : "Je me sens prête, mais avec l'expérience, je pourrai accumuler divers trucs. Il y a des contenus que je me sens toujours moins à l'aise d'enseigner ». Enfin, 17,6 \% des étudiants considèrent qu'ils doivent maitriser davantage les contenus à enseigner $(C M S)$ et 7,8\% souhaiteraient développer des compétences spécifiques pour enseigner aux élèves en difficulté ( $C M S$ et $C P D)$. Selon nous, tous ces commentaires renvoient à la nécessité de travailler les liens entre les $C M S$ et les $C P D$ afin de favoriser le développement des compétences à l'enseignement des mathématiques et de renforcer le SEP.

Au regard du dispositif de formation, les étudiants soulèvent des insatisfactions. Effectivement, 11,8 \% d'entre eux affirment que le travail réalisé pour développer leurs compétences en mathématiques $(C M C$ et $C H M)$ n'a eu aucun effet sur leur formation didactique $(C P D)$ et 33,3\% perçoivent des lacunes à celle-ci. La plus importante concerne le peu de liens entre la formation et la réalité de la classe. Près de $22 \%$ des étudiants considèrent que les cours de didactique sont trop théoriques. L'étudiant ÉL12-2P écrit : "Les cours de didactique devraient être beaucoup plus concrets. Prendre des exemples tirés des cabiers de l'élève et modéliser comment les enseigner. Cela serait plus pertinent que la numération égyptienne ». Enfin, 19,6\% des étudiants affirment que les stages en milieux scolaires s'avèrent la formation la plus pertinente pour apprendre à enseigner (CMS et $C P D)$, comme en témoigne l'étudiant ÉL4-1P : «Je trouve que les stages mont permis de mieux comprendre comment enseigner les mathématiques comparativement aux cours. Les cours ne mont pas vraiment permis de savoir quelle notion enseigner, quand et de quelle manière le faire ». Bien que la majorité d'entre eux reconnaissent la nécessité d'une bonne maitrise des connaissances mathématiques pour l'enseignement $(C S$ et $C P D)$, ceux-ci attribuent cette maitrise plutôt au milieu de pratique. Ces jugements n'étonnent pas, mais démontrent également le peu de liens qu'ils font entre les $C M S$ et les $C P D$.

\section{Discussion}

Plusieurs études ont démontré que les futurs maitres ont des attitudes négatives à l'égard des mathématiques, de leur apprentissage et enseignement (Goasdoué, 2018; Lafortune, 1997; Siety, 2012). Nos fréquences aux différentes questions de l'enquête ont plutôt montré le contraire. En effet, la démarche de développement des compétences dans laquelle les étudiants s'inscrivent permet de prendre conscience de leurs lacunes et de réaliser un travail sur les connaissances mathématiques $(C M C$ et $C H M$ ). Toutefois, la prise de conscience « de la nécessité du travail pour réussir » s'avère une étape fondamentale de cette démarche puisqu'elle va favoriser le développement d'attitudes professionnelles, soit l'importance de maitriser les savoirs mathématiques et les savoirs à enseigner $(C M S)$ et l'ouverture à la formation continue (CMS et $C P D)$, ce qui nourrit le SEP selon Bandura (2007). 
Au regard des commentaires formulés par les étudiants et des taux de réussite à l'examen, cette prise de conscience n'arrive pas assez tôt. Cela peut expliquer, partiellement, le nombre d'étudiants affirmant ne pas avoir amélioré leurs compétences en mathématiques (48,1\%). Une démarche personnelle sérieuse, amorcée dès le début de la formation, est une condition indispensable pour développer des compétences et se sentir prêt à enseigner les mathématiques. Mais elle est aussi tributaire de la capacité à porter un regard critique sur ses propres compétences, dans une perspective de développement professionnel.

Pour plusieurs chercheurs (Batteau, 2020; Bednarz et Proulx, 2009; Coulange et Robert, 2015; Mangiante-Orsola, 2012; Roditi, 2018; Tambone, 2010), les mathématiques de la pratique professionnelle nécessiteraient une articulation des dimensions mathématique, didactique et pédagogique, car dans la pratique enseignante, celles-ci sont imbriquées et souvent mobilisées en simultané. Notre étude a permis de faire ressortir la nécessité et la pertinence de cette articulation dans le déploiement des différents volets du dispositif de formation à l'enseignement des mathématiques de l'UQAR. Effectivement, la plus grande insatisfaction des étudiants quant au dispositif de formation est le peu de liens entre les formations mathématique, didactique et pratique. L'enquête que nous avons réalisée au même moment auprès des enseignants associés a révélé la même problématique. Ceci génère quelques constats : la difficulté pour les étudiants à transposer le travail didactique à la pratique de classe; la difficulté pour le formateur de rendre évidents les liens entre la formation mathématique, didactique et les pratiques d'enseignement; le rôle des stages dans la formation didactique ainsi qu'une meilleure concertation entre les équipes-cours. Quelles sont les nouvelles actions à poser pour améliorer la formation à l'enseignement des mathématiques?

\section{Conclusion}

Notre étude visait à caractériser la démarche de développement des compétences à l'enseignement des mathématiques et à connaître les impacts du dispositif de formation à l'UQAR. Au terme de cette investigation, nous savons que les étudiants privilégient des mesures de courte durée, caractérisée principalement par de l'exercisation. En leur offrant un contexte de développement des compétences au sein duquel ils doivent entreprendre une démarche personnelle, la majorité des étudiants qui s'exercent, révisent et s'impliquent parviennent à développer des attitudes favorables et des compétences à l'enseignement des mathématiques. Cette démarche cultive et alimente leur SEP (Bandura, 2007). Ce sont tous des impacts positifs du dispositif de formation.

Par ailleurs, pour assurer une plus grande satisfaction des étudiants quant à la formation didactique, les activités proposées doivent faire écho aux pratiques de classe afin d'en dégager les connaissances mathématiques et didactiques utiles à leur apprentissage. Ainsi, nous pensons que les enseignants associés doivent être intégrés dans la conception et le développement des programmes de formation initiale à l'enseignement. Les stages de formation pratique doivent être des occasions d'articulation et de collaboration entre formateurs et chercheurs, dans une dynamique d'approche-programme.

À notre avis, un dispositif intégrant les dimensions diagnostique, mathématique, didactique et pratique dans une démarche réflexive favoriserait une telle articulation et collaboration. Conséquemment, pour bonifier le dispositif initial présenté à la figure 1, l'ajout d'un volet pratique aux trois volets existants s'avère essentiel. Effectivement, l'articulation des quatre volets viserait à opérationnaliser dans le 
milieu de pratique, les savoirs mathématiques et didactiques vus en salle de classe universitaire et réciproquement, les enseignements ou les expériences issus du milieu de pratique enrichiraient les savoirs théoriques susceptibles d'être enseignés, favorisant la prise de conscience de la pertinence des contenus mathématiques.

La figure 4 expose l'interaction entre les quatre volets permettant d'assurer une formation reposant sur une réflexion ancrée dans la pratique professionnelle de l'enseignant (Proulx et al., 2012), celle-ci étant indispensable au développement des compétences à l'enseignement des mathématiques et au SEP.

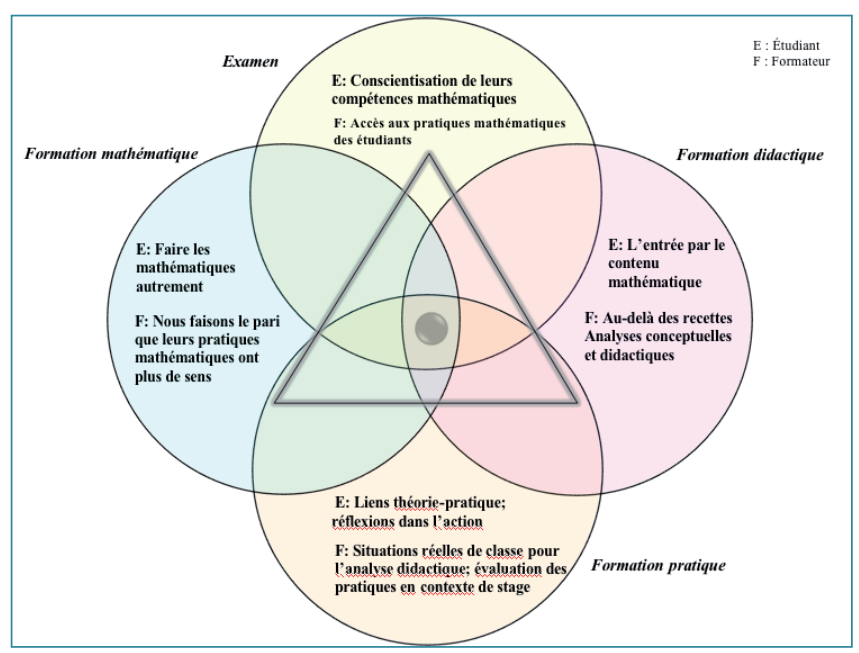

\section{Figure 4}

Dispositif de formation à l'enseignement des mathématiques : articulation des volets et lieux de formation.

Peu de recherches se sont intéressées à cette articulation des formations comme objet d'études (Bednarz, 2012; GREFEM, 2012). En nous interrogeant sur la pertinence du dispositif de l'UQAR, nous avons rendu explicite cette nécessité d'articuler les différents volets de la formation. Toutefois, celle-ci ne peut se réaliser sans la collaboration de tous les intervenants impliqués dans les formations visant le développement des compétences en mathématiques (Bednarz, 2013).

\section{Notes}

1 La forme masculine utilisée dans cet article désigne aussi bien les femmes que les hommes, sans aucune discrimination, dans le seul but d'alléger le texte.

2 Il s'agit d'un examen ayant un double statut : diagnostique et certificatif. L'étudiant doit le réussir avant le début de sa quatrième année de formation, autrement il est suspendu du programme jusqu'à la réussite de l'examen.

3 Nous faisons référence aux connaissances de Loewenberg Ball, Thames et Phelps (2008) et utilisons les acronymes apparaissant dans la figure 2 .

4 La codification des étudiants s'est effectuée ainsi : la lettre É pour étudiant, suivi de la lettre L ou R pour les groupes de Lévis ou Rimouski, ainsi qu'un numéro; après le tiret, le nombre et la lettre $\mathrm{P}$ indiquent le nombre de passations à l'examen effectuées par l'étudiant 


\section{Références}

Adihou, A. et Arsenault, C. (2012). Dispositif de formation mathématique pour les enseignants du primaire : choix, caractéristiques, résultats et effets. Dans J. Proulx, C. Corriveau et H. Squalli (dir.), Formation mathématique pour l'enseignement des mathématiques : pratiques, orientations et recherches (p. 225-253). Québec, QC : Presses de l'Université du Québec.

Adihou, A., Arsenault, C. et Marchand, P. (2012). Dispositif de formation mathématique pour les futurs maitres. Dans J.-L. Dorier et S. Coutat (dir.), Enseignement des mathématiques et contrat social : enjeux et défis pour le 21e siècle-Actes du colloque EMF2012 (p. 260-279). Repéré à http://emf.unige.ch/files/3614/5320/1461/EMF2012GT2ADIHOU.pdf

Bandura, A. (2007). Auto-efficacité : le sentiment d'efficacité personnelle. Paris : De Boeck Université.

Batteau, V. (2020). Évolution des pratiques d'une enseignante d'école primaire lors d'un dispositif de formation et de recherche en mathématiques. Recherches en didactique des mathématiques, 40(1), 13-53.

Bednarz, N. (2012). Formation mathématique des enseignants : état des lieux, questions et perspectives. Dans J. Proulx, C. Corriveau et H. Squalli (dir.), Formation mathématique pour l'enseignement des mathématiques : pratiques, orientations et recherches (p. 13-54). Québec, QC : Presses de l'Université du Québec.

Bednarz, N. (dir.). (2013). Recherche collaborative et pratique enseignante : regarder ensemble autrement. Paris : L'Harmattan.

Bednarz, N. et Proulx, J. (2009). Knowing and using mathematics in teaching: conceptual and epistemological clarifications. For Learning of Mathematics, 29(3), 11-17.

Brousseau, G. (1998). Théorie des situations didactiques : didactique des mathématiques 1970-1990. Grenoble : La pensée sauvage.

Carré, P. (2009). Avant-propos. Dans G. Lameul, A. Jézégou, A.-F. Trollat (dir.), Articuler dispositifs de formation et dispositions des apprenants (p. 9-11). Lyon : Chronique sociale.

Clivaz, S. (2011). Des mathématiques pour enseigner : analyse de l'influence des connaissances mathématiques d'enseignants vaudois sur leur enseignement des mathématiques à l'école primaire (Thèse de doctorat, Université de Genève). Repéré à http://archive-ouverte.unige.ch/unige: 17047

Clivaz, S. (2016). Connaissances mathématiques des enseignants et enseignement de l'algorithme de la multiplication. Recherches en didactique des mathématiques, 36(2), 231-261. Repéré à https://revue-rdm.com/2016/connaissances-mathematiques-des/

Conne, F. (1989). L'articulation des contenus et des moyens et leur double nature mathématique et didactique dans l'enseignement des mathématiques et son évolution. Bulletin de l'association mathématique du Québec (AMQ), 29(3), 814. Repéré à https://halshs.archives-ouvertes.fr/halshs-01986893/document

Coulange, L. et Robert, A. (2015). Les mathématiques dans les activités du professeur : conséquences pour la formation. Dans L. Theis (dir.), Actes du $6^{e}$ colloque Espace mathématique francophone, "Pluralités culturelles et universalité des mathe冈matiques : enjeux et perspectives pour leur enseignement et leur apprentissage » (p. 81-94). Repéré à http://emf.unige.ch/files/6214/6400/7494/EMF2015GT1COULANGE.pdf

DeBlois, L. (2010). Développer une formation à l'enseignement : trois entrées possibles. Dans J. Proulx et L. Gattuso (dir.), Formation des enseignants en mathématiques : tendances et perspectives actuelles (p. 31-37). Sherbrooke, QC : CRP.

Goasdoué, R. (2018). Regards psychologiques sur les apprentissages mathématiques. Dans J.-L. Dorier, G. Gueudet, M.L. Peltier, A. Robert et É. Roditi (dir.), Enseigner les mathématiques : didactique et enjeux de l'apprentissage (p. 165-212). Paris : Belin.

GREFEM. (2012). Formation didactique articulée à la pratique enseignante : illustrations et conceptualisations. Dans J.-L. Dorier et S. Coutat (dir.), Actes du 5 e colloque Espace mathématique francophone, "Enseignement des mathématiques et contrat social : enjeux et défis pour le 21e siècle» (p. 348-361). Repéré à http://emf.unige.ch/files/1614/5320/1511/EMF2012GT2GREFEM.pdf

Lafortune, L. (1997). Dimension affective en mathématiques. Bruxelles : De Boeck. 
Lajoie, C., Maheux, J.-F., Marchand, P., Adihou, A. et Bisson, C. (2012). Le jeu de rôles comme approche de formation à l'enseignement des mathématiques. Quels choix? Pour quelles intentions? Pour quelle formation? Dans Groupe de didactique des mathématiques (dir.), Colloque du GDM 2012 (p. 48-56). Repéré à https://www.dropbox.com/s/xcvmy42wy0j9zhz/2012\%20GDM\%20Actes.pdf

Lajoie, C. et Tempier, F. (dir.). (2019). Numéro spécial sur les dispositifs de formation à l'enseignement des mathématiques. Canadian Journal of Science, Mathematics and Technology Education, 19(2), 83-216.

Loewenberg Ball, D., Thames, M. H. et Phelps, G. (2008). Content knowledge for teaching: What makes it special? Journal of Teacher Education, 59(5), 389-407. http://dx.doi.org/10.1177/0022487108324554

Mangiante-Orsola, C. (2012). Une étude de la cohérence en germe dans les pratiques de professeurs des écoles en formation initiale puis débutants. Recherches en didactique des mathématiques, 32(3), 289-331. Repéré à https://revue-rdm.com/2012/une-etude-de-la-coherence-en-germe/

Miles, M. B. et Huberman, A. M. (2007). Analyse des données qualitatives (2éd.). Bruxelles : De Boeck Université.

Ministère de l'Éducation (MEQ). (2001). La formation à l'enseignement. Les orientations. Les compétences professionnelles. Repéré à http://www.education.gouv.qc.ca/fileadmin/site web/documents/reseau/formation titularisation/formation enseignement orientations EN.pdf

Mucchielli, R. (2006). L'analyse de contenu des documents et des communications (9e éd.). Issy-les-Moulineaux : ESF.

Proulx, J. (2019). Faire vivre une formation à l'enseignement des mathématiques par résolution de problème : le cas du cours «Didactique des mathématiques II et laboratoire » à l'Université du Québec à Montréal. Canadian Journal of Science, Mathematics and Technology Education, 19(2), 120-142. http://dx.doi.org/10.1007/s42330-019-00044-y

Proulx, J., Corriveau, C. et Squalli, H. (dir.). (2012). Formation mathématique pour l'enseignement des mathématiques : pratiques, orientations et recherches. Québec, QC : Presses de l'Université du Québec.

Robert, A. (2008). La double approche didactique et ergonomique pour l'analyse des pratiques d'enseignants de mathématiques. Dans F. Vanderbrouck (dir.), La classe de mathématiques : activités des élèves et pratiques des enseignants (p. 59-68). Toulouse : Octares.

Roditi, É. (2018). Pratique enseignante et didactique des mathématiques. Dans J.-L. Dorier, G. Gueudet, M.-L. Peltier, A. Robert et É. Roditi (dir.), Enseigner les mathématiques : didactique et enjeux de l'apprentissage (p. 89-100). Paris : Belin.

Shulman, L. S. (1986). Those who understand: Knowledge growth in teaching. Educational Researcher, 15(2), 4-14. http://dx.doi.org/10.3102\%2F0013189X015002004

Shulman, L. S. (1987). Knowledge and teaching: foundations of the new reform. Harvard Educational Review, 57(1), 1-23. http://dx.doi.org/10.17763/haer.57.1.j463w79r56455411

Siety, A. (2012). Qui a peur des mathématiques? Paris : Denoël.

Tambone, J. (2010). Un dispositif de recherche pour observer les pratiques enseignantes : l'observation des maitres spécialisés en adaptation scolaire. Recherches en didactique des mathématiques, 30(3), 275-315. Repéré à https://revue-rdm.com/2010/un-dispositif-de-recherche-pour/ 


\section{Annexe 1}

Questionnaire destiné aux étudiants de quatrième année de formation

\begin{tabular}{|c|c|}
\hline \multicolumn{2}{|r|}{ Questions à degré d'accord } \\
\hline $1-$ & Les mathématiques me passionnent. \\
\hline $2-$ & Faire des mathématiques m’angoisse. \\
\hline $3-$ & On doit avoir une solide base en mathématique pour pouvoir les enseigner au primaire. \\
\hline $4-$ & Enseigner les mathématiques me fait peur. \\
\hline $5-$ & Quand j'étais élève, j'avais de la difficulté en mathématiques. \\
\hline $6-$ & Je souffre de « mathophobie » (peur excessive des mathématiques). \\
\hline $7-$ & Quand j'enseignerai les mathématiques, je ne devrai pas me tromper devant les élèves. \\
\hline $8-$ & Je poursuivrai ma formation mathématique en formation continue. \\
\hline $9-$ & Dans mes stages, j'ai eu de la difficulté à enseigner certains concepts mathématiques. \\
\hline $10-$ & II m’arrive de ne pas être capable de résoudre un problème en mathématiques. \\
\hline $11-$ & $\begin{array}{l}\text { La démarche de développement des compétences en mathématiques à I'UQAR m'a permis d'améliorer mes } \\
\text { connaissances et compétences en mathématiques. }\end{array}$ \\
\hline $12-$ & $\begin{array}{l}\text { Un étudiant moins bon en mathématique peut enseigner les mathématiques correctement en se basant sur les guides } \\
\text { d'enseignement. }\end{array}$ \\
\hline $13-$ & Lorsque je terminerai mes études, je serai un excellent enseignant en mathématiques. \\
\hline $14-$ & Un ancien élève faible en mathématiques peut devenir un bon enseignant en mathématiques. \\
\hline $15-$ & Mon attitude envers les mathématiques peut influencer celle des élèves. \\
\hline $16-$ & La formation mathématique est importante dans ma formation à l'enseignement. \\
\hline $17-$ & La formation mathématique reçue à l'UQAR a changé positivement mon attitude envers les mathématiques. \\
\hline $18-$ & $\begin{array}{l}\text { La formation mathématique reçue à I'UQAR m'a aidé à maitriser des contenus mathématiques et à les enseigner en } \\
\text { stage. }\end{array}$ \\
\hline $19-$ & Je suis satisfait de la formation mathématique que j'ai reçue jusqu'ici. \\
\hline $20-$ & $\begin{array}{l}\text { Je me sens confiant d'enseigner les mathématiques parce que je maitrise les contenus reliés à : a) I'algèbre; b) la } \\
\text { résolution de problèmes; c) la géométrie; d) la probabilité et la statistique; e) l'ensemble des nombres et des opérations. }\end{array}$ \\
\hline \multicolumn{2}{|r|}{ Questions à court développement } \\
\hline $21 \mathrm{a}-$ & $\begin{array}{l}\text { Avez-vous bénéficié des mesures d'aide suivantes : (cochez la ou les cases pertinentes) } \\
\text { Ateliers en mathématiques - nombre d'atelier(s) suivi(s); Aide offerte par le CAR; Consultation de votre examen; Cours } \\
\text { de mathématiques : SCE - } 108 \text { - } 05 \text { Ensemble de nombres et opérations sur les nombres; SCE - } 109 \text { - } 05 \text { Algèbre et } \\
\text { résolution; SCE - } 110 \text { - } 05 \text { Géométrie et probabilité; SCE - } 111 \text { - } 06 \text { Savoirs mathématiques; Autre(s) mesure(s), précisez }\end{array}$ \\
\hline $21 b-$ & $\begin{array}{l}\text { Indiquez votre degré de satisfaction par rapport aux mesures d'aide offertes à I'UQAR (4 - très satisfait; } 3 \text { - plutôt } \\
\text { satisfait; } 2 \text { - plutôt insatisfait; } 1 \text { - très insatisfait; } 0 \text { - ne s'applique pas) } \\
\text { Atelier en mathématiques; Cours de mathématiques : SCE - } 108 \text { - } 05 \text { Ensemble de nombres et opérations sur les } \\
\text { nombres; SCE - } 109 \text { - } 05 \text { Algèbre et résolution; SCE - } 110 \text { - } 05 \text { Géométrie et probabilité; SCE - } 111 \text { - } 06 \text { Savoirs } \\
\text { mathématiques }\end{array}$ \\
\hline
\end{tabular}


21c - Si vous avez bénéficié d'autres mesures d'aide que celles mises en place par I'UQAR, expliquez brièvement les raisons qui vous ont amené à faire ce choix.

22 - Vous avez reçu un bilan diagnostique lors de la passation de l'examen de compétences en mathématiques.

a) Quelle est l'importance accordée à ce bilan dans l'orientation de votre démarche de développement de vos compétences en mathématiques?

b) Quelles ont été les étapes de votre démarche afin de prendre en charge vos difficultés en mathématiques?

23 - Quelles autres mesures d'aide que celles déjà prévues auraient pu être mises à votre disposition pour vous aider à améliorer vos compétences en mathématiques tout au cours de votre baccalauréat?

24 - Que retenez-vous du travail accompli en mathématiques au cours de votre baccalauréat? Ce travail a-t-il eu un effet sur votre formation didactique?

25 - Si vous aviez des conseils à donner aux étudiants de première année pour l'amélioration de leurs compétences en mathématiques, que leur diriez-vous?

26 - Dans quelle mesure vous sentez-vous prêt à enseigner les mathématiques au préscolaire-primaire? Si vous ne vous sentez pas prêt, que vous manque-t-il?

\section{Pour citer cet article}

Adihou, A. et Arsenault, C. (2020). Formation à l'enseignement des mathématiques : opérationnalisation et regard des étudiants sur un dispositif de formation. Formation et profession, 28(3), 64-80. http://dx.doi.org/10.18162/fp.2020.511 